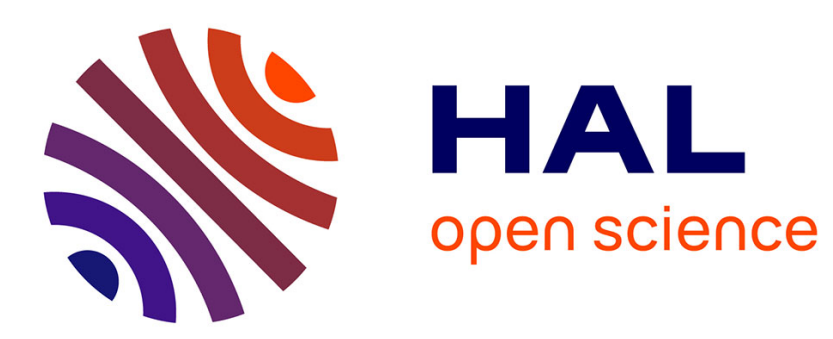

\title{
Étude des surfaces et interfaces à l'aide de la nouvelle génération de synchrotrons
}

\author{
G. Margaritondo
}

\section{To cite this version:}

G. Margaritondo. Étude des surfaces et interfaces à l'aide de la nouvelle génération de synchrotrons. Journal de Physique IV Proceedings, 1997, 07 (C6), pp.C6-31-C6-40. 10.1051/jp4:1997603 . jpa00255702

\section{HAL Id: jpa-00255702 https://hal.science/jpa-00255702}

Submitted on 1 Jan 1997

HAL is a multi-disciplinary open access archive for the deposit and dissemination of scientific research documents, whether they are published or not. The documents may come from teaching and research institutions in France or abroad, or from public or private research centers.
L'archive ouverte pluridisciplinaire HAL, est destinée au dépôt et à la diffusion de documents scientifiques de niveau recherche, publiés ou non, émanant des établissements d'enseignement et de recherche français ou étrangers, des laboratoires publics ou privés. 


\title{
Étude des surfaces et interfaces à l’aide de la nouvelle génération de synchrotrons
}

\author{
G. Margaritondo
}

Institut de Physique Appliquée, École Polytechnique Fédérale, 1015 Lausanne, Switzerland, et, Sincrotrone Trieste, Trieste, Italy

\begin{abstract}
Résumé : Deux ans après leur mise en fonction et sur la base de l'ample expérience pratique acquise par des centaines de scientifiques, les sources de rayonnement synchrotron de la troisième génération sont prêtes à être soumises à une analyse critique de leurs performances. Les questions qui s'imposent sont donc les suivantes: peut-on confirmer que la brillance était le bon choix pour les sources de cette nouvelle génération? Peut-on effectivement constatèr l'impact du haut niveau de brillance sur des applications réelles? Nous essayerons de répondre à ces questions dans le domaine spécifique de la physique et chimie des surfaces et interfaces, à l'aide d'une série d'exemples provenant principalement du laboratoire ELETTRA en Italie.
\end{abstract}

Summary: Two years after their commissioning, the third-generation synchrotron source are ripe for a critical analysis of their performances, based on the broad practical experience of hundreds of scientists. The most relevant questions are: can one confirm that high brightness was the best choice for the sources of this new generation? Can one actually see the impact of the high brightness level on real applications? We try to answer these questions in the specific domain of surface and interface chemistry and physics, using a series of examples, primarily provided by the ELETTRA laboratory in Italy.

\section{INTRODUCTION}

La recherche sur les surfaces et les interfaces a grandement bénéficié, à partir des années soixante-dix, des performances avancées des sources de rayonnement synchrotron, notamment en ce qui concerne les expériences se basant sur la photoémission.[1] A leur tour, les performances de ces mêmes sources ont progressivement évolué, et cette évolution a été marquée par trois générations successives d'accélérateurs.

La première ne comprenait que des accélérateurs conçus pour d'autres branches de recherche, et donc bien loin d'être optimisés en vue d'applications comme sources de rayons $X$. La deuxième génération vit l'augmentation du flux de l'émission, par le développement d'accélérateurs spécifiquement conçus pour ces applications.

Ce n'est qu'avec la troisième génération, mise en fonction à partir de la moitié des années quatrevingt-dix, que la brillance a été reconnue comme le paramètre le plus important pour caractériser la performance d'une source de rayonnement synchrotron.[2] Ce paramètre combine le flux aux caractéristiques géométriques de la source: sa dimension et sa divergence angulaire.[2,3]

La base thérique pour le choix de la brillance comme mesure principale de performance est fournie par une des versions du théorème de Liouville.[2,3] Celle-ci prévoit la conservation de la brillance le long d'un système optique idéal, sans pertes. Donc, si l'on souhaite obtenir une brillance élevée à la fin d'une ligne de lumière synchrotron, il est préférable de commencer par une source dotée elle-même d'une brillance élevée. Par ailleurs, la plupart des applications du rayonnement synchrotron - surtout dans la recherche sur les surfaces et les interfaces - exigent une brillance maximale.

Les sources de la troisième génération sont actuellement une réalité: des anneaux d'accumulation tels qu'ELETTRA en Italie, ESRF à Grenoble et ALS et APS aux USA, ainsi que d'autres en Corée, à Taiwan et en Suedde, se distinguent par leur brillance extrême. Celle-ci dépasse de plusieurs ordres de grandeur la génération précédente, grâce à un contrôle extrêmement précis de la géométrie đu faisceau d'électrons dans l'anneau d'accumulation, ainsi qu'à l'utilisation systématique de dispositifs d'émission du type onduleur.

Tous les projets ont atteint et même - dans la majorité des cas - dépassé les performances prévues, donc la question n'est plus de confirmer que celles-ci sont effectivement possibles. De plus, les sources fonctionnent déjà depuis une année ou deux avec une excellente continuité, et sont parfaitement en mesure de servir les utilisateurs externes les plus exigeants. 
Il est donc actuellement possible de se poser la question: peut-on confirmer que la brillance était le meilleur choix pour la nouvelle génération de sources? On peut analyser ce point sur la base de l'expérience pratique de centaines de chercheurs.

Grâce à une série de résultats récents, nous verrons que la haute brillance a réellement et fortement changé les techniques de recherche sur les surfaces et interfaces, en étendant également leurs domaines d'application. Les exemples que nous discuterons concernent en premier lieu la spectroscopie à très haute résolution et la spectromicroscopie, tant à balayage qu'à formation d'images.[4] Ensuite, nous passerons à la spectroscopie en fonction du temps, qui permet de passer des études statiques à celles de la dynamique des réactions chimiques des surfaces et interfaces. [5]

La discussion sera complétée par une analyse des développements prévus pour les années à venir, surtout des projets des premières sources de rayonnement synchrotron de la quatrième génération. A notre avis, celle-ci sera caractérisée par une brillance encore plus élevée, mais avant tout par son extrême cohérence.

\section{PHOTOÉMISSION : DE LA SPECTROSCOPIE À LA SPECTROMICROSCOPIE}

En ce qui concerne la recherche sur les surfaces et les interfaces, la plupart des applications du rayonnement synchrotron sont liées à l'effet photoélectrique. La disponibilité des sources avancées de rayons $\mathrm{X}$ et ultraviolets a permis, à partir des années soixante-dix, le développement de nouvelles techniques telles que la photoémission à résolution angulaire (et l'étude expérimentale des bandes d'énergie), les techniques de type CIS et CFS ("constant initial state" et "constant final state"), les études de phénomènes de résonance et plusieurs autres applications.[1]

Il est donc souhaitable d'utiliser ce domaine spécifique comme échantillon pour analyser l'impact pratique de la haute brillance de la troisième génération de sources: celle-ci, a-t-elle en fait changé de façon importante les applications du rayonnement synchrotron en photoémission?

La Fig. 1 fournit une première réponse positive à cette question: il s'agit de spectres de photoémission du niveau de coeur 3d5/2 d'un cristal de rhodium. Les résultats ont été obtenus par Zacchigna et al.[6], à l'aide de la ligne de photoémission à ultraviolet à vide du laboratoire de troisième génération ELETTRA à Trieste, en Italie. ELETTRA est actuellement, avec l'accélérateur ALS ("Advanced Light Source") à Berkeley, la source de rayons $\mathrm{X}$ mous la plus brillante du monde.

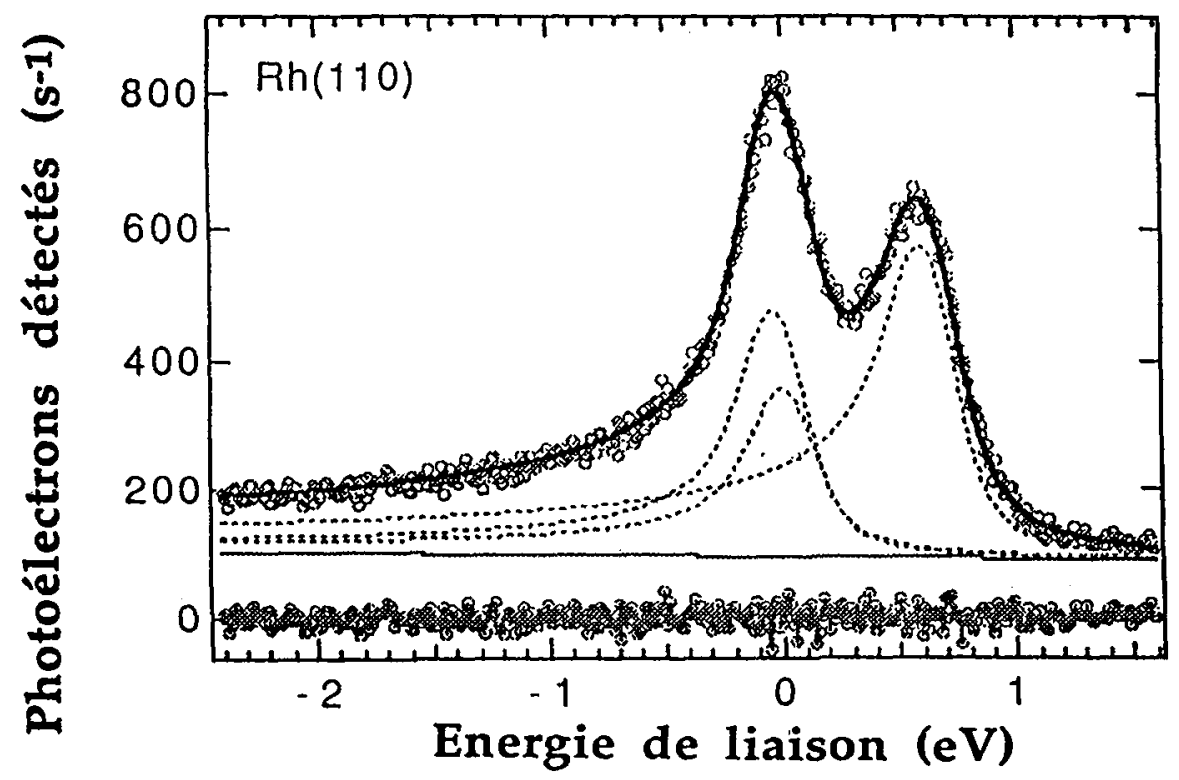

Figure 1 - Spectres de photoémission à très haute résolution du niveau de coeur $3 \mathrm{~d} 5 / 2$ du rhodium, obtenus par la ligne de photoémission dans l'ultraviolet à vide d'ELETTRA par les auteurs de la Réf. 6. 
Ce niveau de brillance permet d'améliorer fortement les performances de la ligne de lumière, et en ce qui concerne le système de photoémission, d'augmenter la résolution angulaire et en énergie, non pas en diminuant mais souvent en augmentant les niveaux de signal. Il est donc possible d'atteindre des performances inédites, mais aussi de mener des expériences très rapides. La Fig. 1 en est un bon exemple.

Celle-ci révèle clairement la structure du spectre $3 \mathrm{~d}_{5 / 2}$, qui comprend deux composantes principales: le pic de gauche est produit par la photoémission des atomes internes du cristal, tandis que celui de droite provient des atomes de la surface du cristal.

L'existence de composantes internes et de surface dans les spectres de ce type était déjà connue, mais les performances de la troisième génération de sources permettent des études pointues qui seraient impossibles autrement. Par exemple, l'analyse de la ligne spectrale à l'aide de l'ordinateur n'est plus nécessaire pour révéler la structure principale à deux pics, qui est évidente à partir des données brutes; on peut donc appliquer cette analyse à l'identification d'autres caractéristiques moins évidentes. C'est le cas, par exemple, du troisième pic de la Fig. 1, qui est causé par des atomes dans une région intermédiaire entre la surface et la partie interne du cristal.

De plus, la même analyse permet finalement de révéler des caractéristiques importantes et, pour la plupart, inconnues précédemment de la structure principale des spectres. On peut noter, par exemple, la différence de forme de ligne entre les composantes de surface et interne de la Fig. 1.

Le pic de surface se révèle plus large en énergie et remarquablement plus asymétrique que celui des atomes internes. L'asymétrie des pics de coeur d'un métal est produite par les électrons libres au niveau de Fermi qui se trouvent dans la même région que l'atome émettant le photoélectron. Le phénomène est lié à de petites pertes d'énergie pendant l'émission des photoélectrons, causées à leur tour par des excitations électron-trou de basse énergie, donc proches du niveau de Fermi.[1] La différence d'asymétrie révèle donc une différence de densité de charge libre à basse énergie, plus élevée pour la surface que pour la partie interne du cristal.

En ce qui concerne la différence générale de largeur de ligne, celle-ci est elle-même produite par une différence de densité de charge libre entre surface et partie interne. Pourtant, la différence n'est plus limitée aux énergies proches du niveau de Fermi. De plus, la différence pourrait partiellement se justifier par des différences du mécanisme d'action des électrons libres, plutôt que simplement par leur densité.

En résumé, dans ce cas les performances de la source permettent d'obtenir une série d'informations supplémentaires intéressantes sur la structure et les propriétés de l'échantillon; ces informations étaient, évidemment, déjà présentes dans les spectres produits par les sources précédentes. Pourtant, les performances limitées de celles-ci en rendaient l'identification et l'utilisation difficile, sinon impossible.

Passons maintenant à une autre amélioration des expériences de photoémission permise par les nouvelles sources. En général, le domaine le plus susceptible de bénéficier des augmentations de brillance est celui de la microscopie. Vu la brillance limitée des sources précédentes, il n'est pas surprenant que les applications de microscopie soient rares en ce qui concerne les rayons $\mathbf{X}$. C'est précisément dans cette direction que la troisième génération de synchrotrons a stimulé une véritable explosion d'activité.

En ce qui concerne la photoémission, on exploite la haute brillance pour mener des expériences à haute résolution latérale. Après quelques années de développement préliminaire, deux approches complémentaires se sont imposées, tous deux combinant des performances de microscopie et de spectroscopie. Les techniques qui en résultent, illustrées par la Fig. 2, sont appelées de "spectromicroscopie".[4]

La première approche est une technique à balayage: le faisceau de rayons $\mathrm{X}$ est focalisé dans une région microscopique de l'échantillon, afin d' obtenir des micrographies photoélectroniques en balayant celui-ci par rapport au faisceau. [4] L'intensité d'une micrographie reproduit le nombre de photoélectrons détectés à chaque point de la surface de l'échantillon, dans la "fenêtre" d'énergie filtrée grâce à un analyseur d'électrons.

Typiquement, la "fenêtre" est sélectionnée dans la région d'émission de photoélectrons d'un niveau de coeur d'un des éléments constituant l'échantillon. L'énergie des photoélectrons contient des informations tant sur l'élément dont ils proviennent que sur l'état chimique de celui-ci. Donc, une micrographie photoélectronique contient des informations chimiques très avancées et utiles.

De plus, on peut utiliser une micrographie photoélectronique pour identifier les régions microscopiques les plus interessantes de l'échantillon, et les illuminer par le faisceau focalisé, obtenant ainsi des spectres de photoémission locaux. En résumé, on a transporté sur le plan microscopique toutes les performances de la spectroscopie de photoémission conventionnelle. Celle-ci est limitée au domaine de 
résolution latérale des dixièmes de millimètres, tandis que la spectromicroscopie peut actuellement atteindre ou même dépasser le dixième de micron.

Quant à la deuxième approche, la résolution latérale n'est pas obtenue en focalisant le faisceau de rayons $\mathrm{X}$, mais en utilisant un système d'optique électronique pour former des images par les photoélectrons. [4] Dans ce cas, l'aspect spectroscopique est lié à l'énergie des photons. Vu la nature du phénomène de photoémission, l'intensité du courant de photoélectrons est proportionnelle à l'absorption des photons. On peut ainsi obtenir des spectres d'absorption de rayons X; la détection et l'analyse des seuils d'absorption permettent d'identifier les éléments correspondants et leurs états chimiques.

\section{Les deux approches à la spectromicroscopie photoélectronique:}

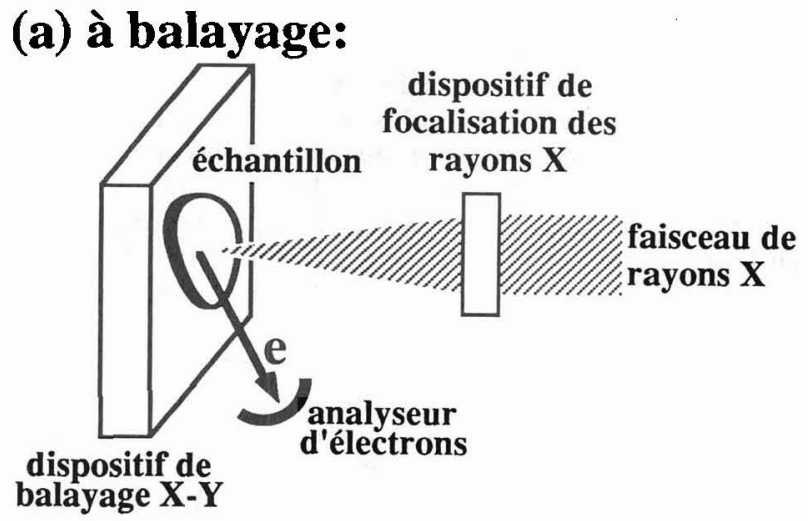

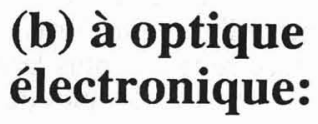

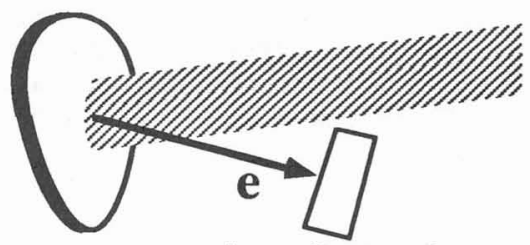

optique électronique

Figure 2 - Les deux approches à la spectromicroscopie photoélectronique (a) à balayage, et (b) à formation d'images par un système d'optique électronique.

Les expériences de spectromicroscopie de ce type analysent premièrement la morphologie du système par des micrographies obtenues grâce à l'optique électronique. Ensuite, des spectres d'absorption sont obtenus dans les régions microscopiques les plus prometteuses. On peut également prendre des micrographies à deux énergies des photons, l'une avant et l'autre après un seuil d'absorption d'un élément donné. La comparaison des deux micrographies, se basant souvent sur la soustraction point par point des intensités, peut révéler avec beaucoup d'efficacité la distribution dans l'espace de l'élément concerné.

Le début des techniques de spectromicroscopie photoélectronique par rayonnement synchrotron se situe à la fin des années quatre-vingt, donc en utilisant des sources de deuxième génération. Pourtant, les expériences étaient assez difficiles et leur domaine d'application beaucoup trop limité. La mise en fonction des sources de troisième génération a révolutionné ce domaine, en rendant les techniques rapides, efficaces et assez facilement applicables à une grande variété de systèmes et de problèmes.

La Fig. 3 en fournit un bon exemple, par des résultats obtenus par Kolmakov et al.[7] en utilisant la ligne de faisceau de Microscopie ESCA à ELETTRA. Cette ligne peut focaliser le faisceau de rayons X jusqu'à atteindre une résolution latérale de 0,1 micron, grâce aux dispositifs appelés lentilles de Fresnel.

Les résultats de la Fig. 3 constituent un très bon exemple des performances actuelles des techniques de spectromicroscopie, en révélant l'influence de la micromorphologie de surface sur la réactivité chimique. La Fig. 3a montre des micrographies du système; celui-ci est un substrat $\mathrm{Si}(111)$ sur lequel on a obtenu par déposition et traitement thermique une première couche mince d'or, suivie par la déposition d'une deuxième couche mince d'argent.

Cette procédure donne lieu à deux phases coexistantes: l'une à deux dimensions (2D) et l'autre à trois dimensions (3D). Le problème à clarifier était le rôle relatif de chaque phase dans la processus de formation de cette interface. 
Les micrographies de la Fig. 3a ont été obtenus en sélectionnant les photoélectrons provenant des niveaux de coeur $\mathrm{Au} 4 \mathrm{f}_{7 / 2}, \mathrm{Si} 2 \mathrm{p}$ and $\mathrm{Ag} 3 \mathrm{~d}_{3 / 2}$, révélant ainsi la distribution des élements correspondants. Ces images montrent la différence d'interaction substrat-couches entre les deux phases 2D et 3D: les agrégats de la phase 3D, riches en or, contiennent moins de Ag et de Si que la phase 2D. De plus, les spectres de photoémission locaux révèlent des différences d'état chimique entre les deux phases.

(a)

\section{SI $2 p$}

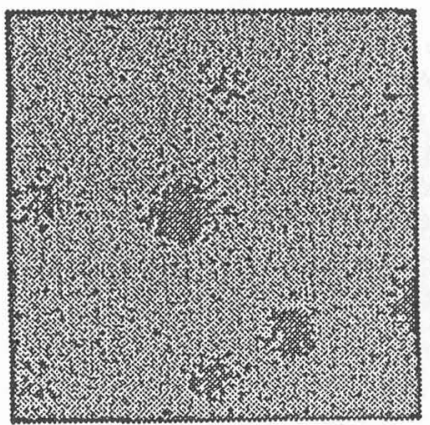

Au $4 f_{7 / 2}$

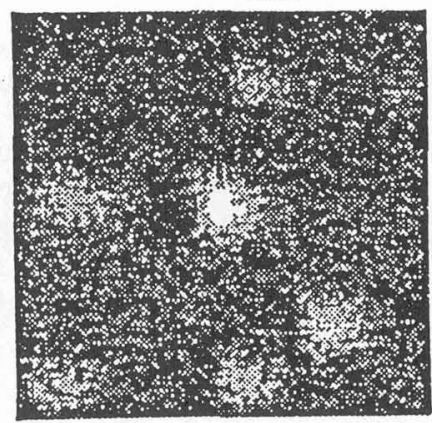

Ag 3d 5/2

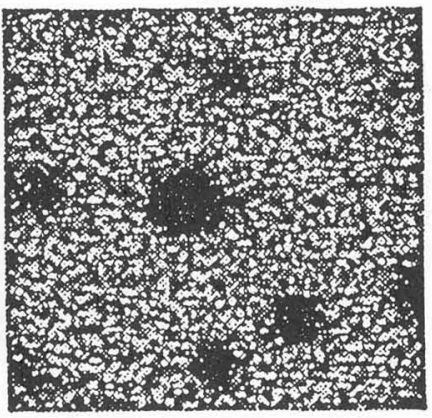

(b)

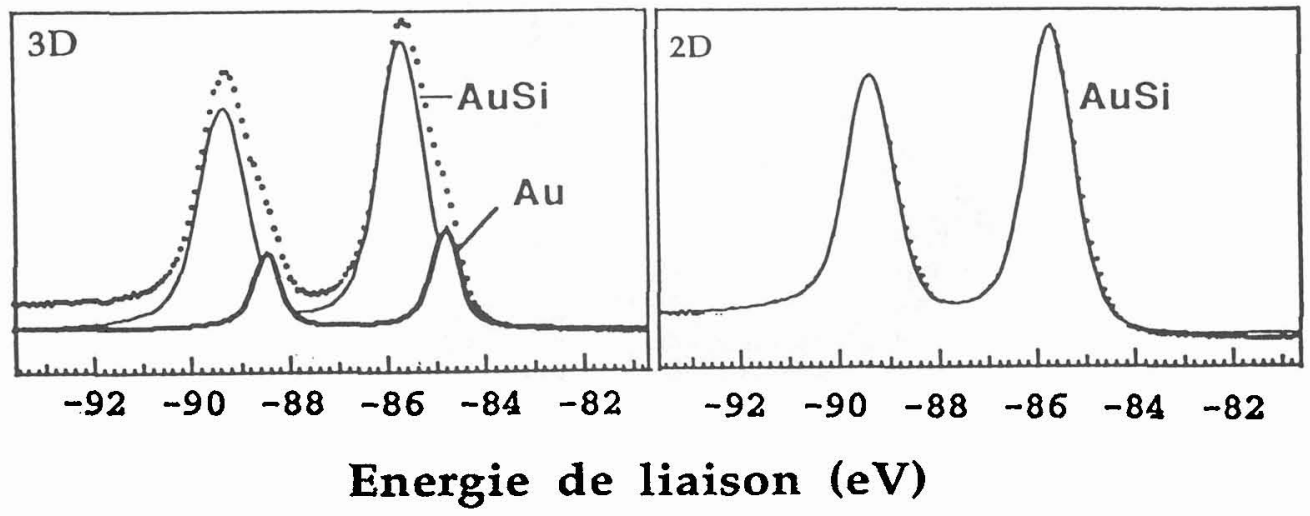

Figure 3 - (a) Micrographies photoélectroniques $\left(6 \times 6 \mu \mathrm{m}^{2}\right)$ montrant la distribution des élements Si, Au et Ag pour un substrat Si(111) recouvert d'abord de $\mathrm{Au}$ et ensuite de Ag. Le système comprend des phases à trois (3D) et à deux (2D) dimensions. (b) Spectres Au4f $7 / 2$ des deux phases. Energie des photons: $492 \mathrm{eV}$. Ces résultats ont été obtenus avec la ligne de microscopie ESCA d'ELETTRA par les auteurs de la Réf. 7.

Plus spécifiquement, les spectres $\mathrm{Au} 4 \mathrm{f}$ de la Fig. 3b montrent que les agrégats 3D contiennent à la fois de l'or métallique et de l'or du système $\mathrm{AuSi}$, tandis que la phase $2 \mathrm{D} \sqrt{3}-\mathrm{Ag}+\mathrm{Au} / \mathrm{Si}$ ne contient que de l'or du type AuSi. L'état chimique local de l'or détermine la dynamique de l'interaction entre or et argent: la présence d'or métallique dans les agrégats 3D justifie leur plus grande réactivité par rapport au reste du système.[7] 
La Fig. 3 n'est qu'un exemple parmi une grande série de données, obtenues tant sur ELETTRA que dans d'autres laboratoires. La qualité de ces résultats est telle que la résolution latérale devient progressivement une necessité en photoémission, sauf pour les techniques dont le niveau de signal n'est pas encore suffisant pour la spectromicroscopie, notamment la spectroscopie de haute résolution $(<200$ $300 \mathrm{meV}$ ) et celle en fonction du temps.

Il faut préciser que la résolution latérale, ayant atteint des valeurs comparables aux dimensions des cellules, rend actuellement possible le transfert des techniques de photoémission, développées à l'origine pour l'analyse des matériaux, au domaine de la biologie. On peut noter, par exemple, les expériences d'analyse microchimique par spectromicroscopie de photoémission des cultures de cellules du cerveau, par G. De Stasio et ses collaborateurs [8]; la Fig. 4 en fournit un exemple.

\section{LE DOMAINE DU TEMPS : CE N'EST QU'UN DÉBUT, MAIS...}

L'évolution dynamique des surfaces et des interfaces est peut-être le sujet le plus intéressant de ce domaine de recherche. Malheureusement, les moyens expérimentaux sont très limités. Jusqu'à récemment, par exemple, la prise de chaque spectre de photoémission nécessitait, quelques minutes, ce qui rendait presque impossibles les études en fonction du temps.

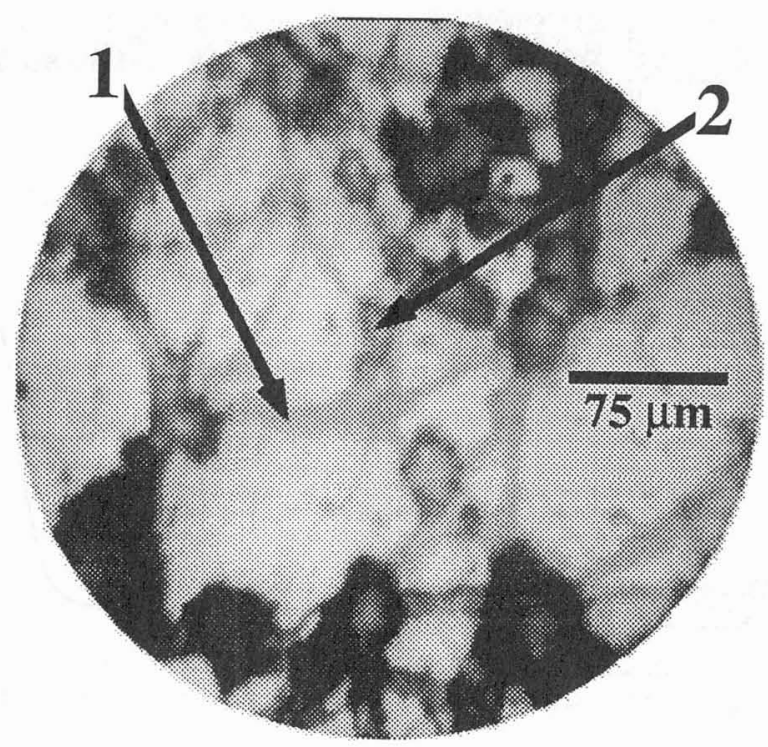

Figure 4 - Exemple (De Stasio et al., Réf. [8]) des applications de la spectromicroscopie photoélectronique en biologie: micrographie d'un réseau de cellules du cerveau. Les points 1 et 2 indiquent [8] les parties de l'échantillon qui ont été analysée par spectroscopie, afin d'étudier les effet de la contamination du réseau par le cobalt.

La brillance élevée d'ELETTRA et, en général, des sources de la troisième génération, a permis [5] de raccourcir le temps de mesure à quelques secondes par spectre, et des améliorations de cette performance sont déjà envisagées, la cible finale se situant à 10-100 millisecondes. La rapidité de prise de données est déjà suffisante pour des études en fonction du temps.

Actuellement, le système expérimental le plus avancé pour des expériences de ce type est la ligne SuperESCA d'ELETTRA, consacrée aux expériences de science des surfaces et interfaces par photoémission en fonction du temps. [5] La ligne est utilisée par un très grand nombre de scientifiques, qui y trouvent enfin un instrument pour dépasser les limites des études statiques et attaquer les problèmes de la dynamique des réactions. 
Les résultats de la Fig. 5 montrent [5] un exemple d'expérience sur la ligne SuperESCA. On peut voir l'évolution en fonction du temps du spectre de photoémission du niveau de coeur O1s pour une surface $\mathrm{Rh}(110)$, exposée à une pression partielle d'oxygène de l'ordre de $5 \times 10^{-9} \mathrm{mbar}$, à la température ambiante.

La figure ne montre qu'une petite partie de la série complète des 80 spectres produits par cette expérience; ceux-ci révèlent l'évolution du système toutes les 12 secondes, et sont en mesure de détecter des changements d'épaisseur de l'ordre de 0,02 monocouche.

Les résultats constituent un excellent exemple de la différence entre les information "statique" des études conventionnelles et la richesse en informations intermédiaires fournies par la séquence de spectres. Une première composante est visible à l'énergie de liaison de 529,65 $\pm 0,05 \mathrm{eV}$; il s'agit de la seule composante spectrale jusqu'à une épaisseur de 0,35 monocouche. Ensuite, l'intensité de cette composante tend à diminuer, et une deuxième composante peut être observée à l'énergie de liaison de 530,25 $\pm 0,05$ $\mathrm{eV}$. Pour des épaisseurs dépassant 0,8 monocouche, seule la deuxième composante peut être observée.

Ces résultats peuvent être interprétés en faisant l'hypothèse que l'adsorption a lieu d'abord dans un long site atomique "de pont", et ensuite dans des sites de symétrie triangulaire de la surface (110). Le rôle dominant du deuxième type de site pour des épaisseurs dépassant 0,35 monocouche est justifié par les interactions oxygène-oxygène, dont l'importance augmente avec l'épaisseur de la couche absorbée. Des atomes d'oxygène adsorbés à l'origine dans le site de pont sont ainsi déplacés vers le site de symétrie triangulaire.

La grande envergure des applications de cette approche est facile à réaliser. Celles-ci comprennent, par exemple, l'analyse des relations entre la vitesse des phénomènes d'adsorption et les changements de la surface, concernant en particulier l'épaisseur équivalente, les sites et les changements de ceux-ci. Il faut noter que d'autres techniques sont très difficiles à utiliser pour s'attaquer à des problèmes comme celui de la Fig. 5: les techniques de spectroscopie des vibrations sont difficiles à appliquer aux couches adsorbées de caractère atomique; d'autres approches, notamment les mesures du coefficient d'adsorption, ne peuvent fournir que des informations génériques, sans clarifier les propriétés de chaque phase chimique.

\section{LE FUTUR : ARRIVÉE DE LA COHÉRENCE}

Notre but n'est évidemment pas de présenter une revue complète des résultats produits par les sources de la troisième génération. Les résultats que nous avons brièvement discutés ont été choisis pour fournir, sur une base empirique, l'évidence claire que le choix stratégique d'augmenter la brillance se traduit d'ores et déjà en performances beaucoup plus avancées, surtout en ce qui concerne les études des surfaces et des interfaces.

La question du choix de la brillance en tant que paramètre de préférence n'est pas académique: encore récemment, des polémiques ont eu lieu sur ce point. Prenons par exemple les propositions de nouvelles sources, qui devraient dépasser les performances d'ELETTRA ou de ALS.

Le projet SLS (Source de Lumière Suisse), récemment développé par l'Institut Paul-Scherrer de Villigen, propose en effet de dépasser la brillance de la troisième génération d'au moins un facteur 5 , grâce à des améliorations révolutionnaires, notamment dans le domaine du réseau d'aimants de l'accélérateur.[9] Le projet SLS se situera ainsi clairement dans une nouvelle - quatrième génération de sources.

D'autre part, un projet pour une nouvelle source - appelé "Soleil" - a été développé en France. Dans sa première version, proposée par le laboratoire LURE d'Orsay, il ne prévoyait pas une augmentation de brillance comme cible prioritaire. Après la présentation de la première version, d'autres propositions ont été développées pour le même projet. En particulier, un groupe de travail présidé par M. le Prof. Georges Charpak a présenté une version se basant clairement sur le choix de la brillance comme paramètre clé.[10]

Suite à de longues polémiques, il existe actuellement une convergence d'opinions en ce qui concerne le projet Soleil: comme pour SLS, la brillance est choisie comme le paramètre le plus important.

A notre avis, ce choix est pleinement justifié. Tout d'abord, par les nombreux résultats révélant les avantages de la haute brillance, ceux présentés par cet article n'en constituant qu'une mince fraction. Mais il existe une raison peut-être plus fondamentale encore.

La brillance des sources de rayonnement synchrotron a pu être augmentée principalement grâce à deux facteurs. Le premier est l'utilisation de dispositifs magnétiques spéciaux pour l'émission de rayons X: les onduleurs et les "wigglers". Lé second est l'amélioration des caractéristiques géométriques du faisceau d'électrons circulant dans l'anneau d'accumulation. 


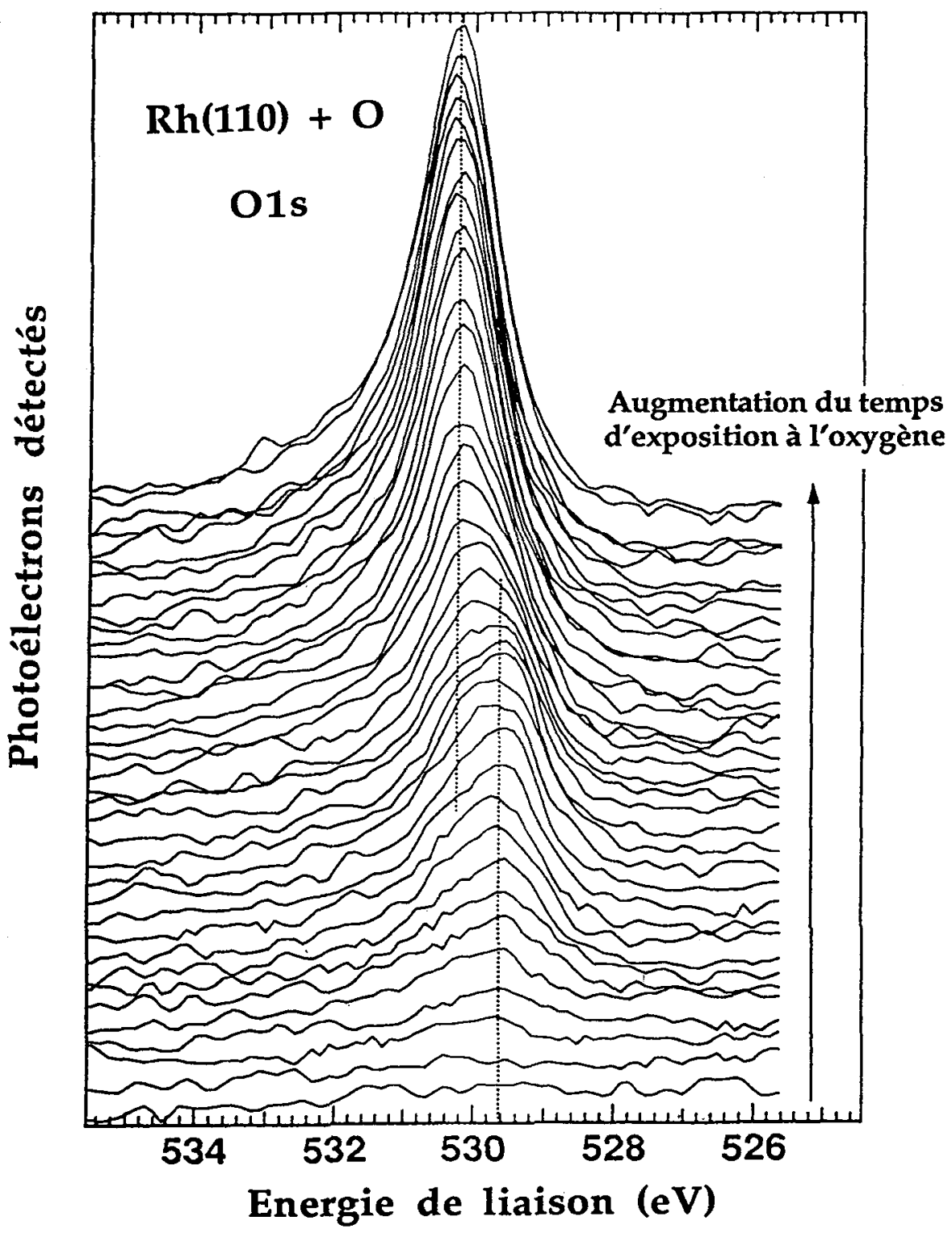

Figure 5 - Série de spectres de phoțómission du niveau de coeur O1s, obtenus en utilisant la ligne SuperESCA, d'ELETIRA par les auteurs de la Réf. 5. Ayant commencé notre recherche sur ce type de spectres quand une seule courbe exigeait deux jours de prise de données, nous trouvons absolument êtonnant que les spectres montrés ici puissent être obtenus en quelques secondes: c'est une des meilleures preuves des avantages de la haute brillance. 
Le point important est que l'augmentation de brillance liée à l'amélioration des conditions géométriques de l'émission (dimension de la source et divergence angulaire) implique également un très haut niveau de cohérence.

Il existe deux types de cohérence: longitudinale et latérale. La cohérence longitudinale est déterminée par la largeur de la bande spectrale d'émission. La brillance élevée de la source permet d'améliorer l'efficacité des monochromateurs dans leur action de filtre, et donc d'augmenter la cohérence longitudinale en diminuant la bande spectrale, sans pourtant diminuer l'intensité à des niveaux inacceptables.

La cohérence latérale est déterminée par les caractéristiques géométriques de la source, c'est-à-đire par les mêmes caractéristiques qui déterminent la brillance: dimension de la surface émettrice et divergence angulaire. La brillance élevée des nouvelles sources de rayonnement synchrotron correspond donc aussi à une haute cohérence latérale.

ELETTRA (Fig. 6) possède d'ores et déjà presque 100\% de cohérence latérale pour des énergies de photons jusqu'à $10 \mathrm{eV}$, et un remarquable niveau de cohérence jusqu'à 1-10 keV. La source suisse SLS permettra d'obtenir $100 \%$ de cohérence latérale jusqu'à $100 \mathrm{eV}$ ou plus. Les nouvelles versions du projet Soleil visent le même résultat.

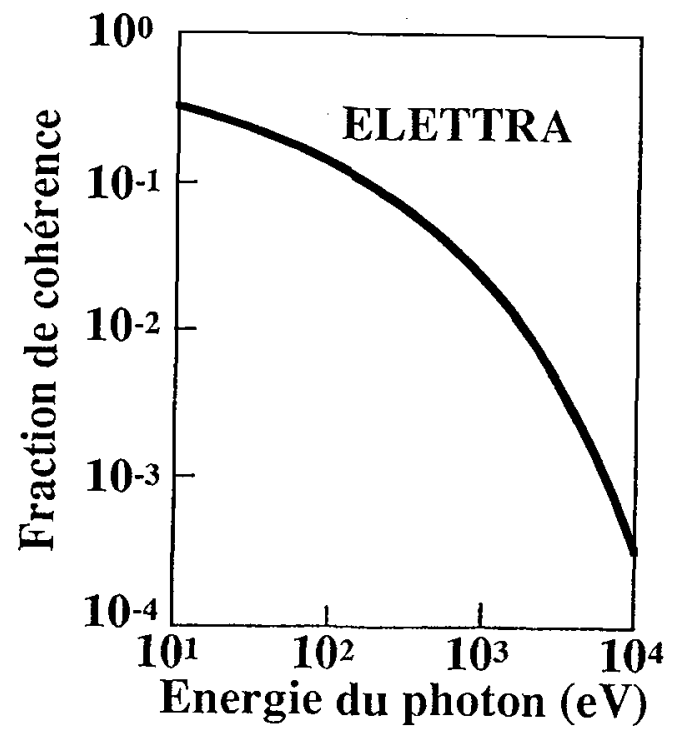

Figure 6 - Fraction de cohérence (latérale) de la source ELETTRA en fonction de l'énergie du photon.

Brillance élevée et cohérence sont deux des caractéristiques importantes des sources lasers. A certains points de vue, les sources de rayonnement synchrotron de la troisième (et encore plus de la quatrième) génération sont donc semblables à des lasers.

Les applications de ces niveaux de cohérence sont encore très limitées, même si des résultats intéressants ont été obtenus, notamment en microscopie et holographie. La rareté des applications de la 
cohérence est assez compréhensible: les chercheurs utilisant les rayons $\mathbf{X}$ n'ont jamais eu de sources cohérentes, donc pour la plupart ils n'ont jamais pris en considération les techniques qui pourraient exploiter un rayonnement à haute cohérence.

Mais cette situation va, sans doute, changer très rapidement. A notre avis, tout comme le passage de la deuxième à la troisième génération de sources a été marqué par le changement de priorité du flux à la brillance, le passage à la quatrième génération remplacera celle-ci par la cohérence.

\section{Remerciements}

Les résultats utilisés pour cet article ont été obtenus par les auteurs des références 5-7. Nous remercions le Fonds National Suisse de la Recherche Scientifique, l'Ecole Polytechnique Fédérale de Lausanne et la société Sincrotrone Trieste SCpA pour le financement de nos activités dans ce domaine.

\section{Références}

[1] G. Margaritondo: Introduction to Synchrotron Radiation (Oxford University Press, New York, 1988).

[2] G. Margaritondo, Riv. Nuovo Cimento 18 (1995) 1-24.

[3] G. Margaritondo, J. Synchr. Rad. 2 (1995) 148-154.

[4] G. Margaritondo et F. Cerrina, Nucl. Instr. Meth. A291 (1990) 26-35 (1990).

[5] A. Baraldi, M. Barnaba, B. Brena, D. Cocco, G. Comelli, S. Lizzit, G. Paolucci and R. Rosei, J. Electron Spectr. 76, 145 (1995); G. Comelli, A. Baraldi, S. Lizzit, G. Paolucci et R. Rosei, en cours de publication.

[6] M. Zacchigna, C. Astaldi, K. C. Prince, M. Sastry, C. Comicioli, M. Evans, R. Rosei, C. Quaresima, C. Ottaviani, C. Crotti, M. Matteucci et P. Perfetti, en cours de publication.

[7] A. Kolmakov, L. Kovac, M. Günther, L. Casalis, L. Gregoratti, M. Marsi et M. Kiskinova, en cours de publication.

[8] Gelsomina De Stasio, F. Cerrina, B. P. Tonner, D. Mercanti et G. Margaritondo, Life Chemistry Reports 11 (1994) 79-90; Gelsomina De Stasio, Delio Mercanti, M. Teresa Ciotti, T. C. Droubay, P. Perfetti, G. Margaritondo and B. P. Tonner, J. Phys. D29, 259 (1996).

[9] H. J. Weyer and G. Margaritondo, Synchrotron Radiation News 8 (1995) 19-20.

[10] P. Soukiassian et G. Margaritondo, Le Monde (11 Novembre 1994), 2. 